\title{
Anti-lactoferrin antibodies and other types of ANCA in ulcerative colitis, primary sclerosing cholangitis, and Crohn's disease
}

\author{
E Peen, S Almer, G Bodemar, B-O Rydén, C Sjölin, K Tejle, T Skogh
}

\begin{abstract}
Fifty two serum samples from patients with Crohn's disease, 24 from patients with ulcerative colitis, and 12 from patients with primary sclerosing cholangitis were analysed for the presence of anti-neutrophil cytoplasm antibodies (ANCA) of IgG and IgA class by means of enzyme linked immunosorbent assays using lactoferrin, myeloperoxidase, and antigen extracted from azurophil granules, ' $\alpha$ antigen' (that is, an antigen preparation containing proteinase 3) as substrates. A high frequency of positive tests for IgG anti-lactoferrin antibodies was found in sera from patients with ulcerative colitis $(50 \%)$ and primary sclerosing cholangitis (50\%). In Crohn's disease only 4 of $52(8 \%)$ sera had anti-lactoferrin antibodies - in all four instances the sera belonged to patients with disease involving the colon. All patients with sclerosing cholangitis and positive tests for anti-lactoferrin had ulcerative colitis. Low levels of IgG antibodies against myeloperoxidase or $\alpha$ antigen were also found occasionally in sera from patients with ulcerative colitis and primary sclerosing cholangitis. IgA antibodies directed against lactoferrin and $\alpha$ antigen (but not myeloperoxidase) were seen in all three conditions.

(Gut 1993; 34: 56-62)
\end{abstract}

Since the description of anti-neutrophil cytoplasm antibodies (ANCA) in active Wegener's granulomatosis, ' the interest in anti-granulocyte antibodies has increased vastly. ANCA is now recognised as a family of autoantibodies directed against cytoplasmic antigens, mainly lysosomal enzymes, in polymorphonuclear neutrophil leukocytes (PMNL). ${ }^{2}$ In Wegener's granulomatosis, ANCA are typically directed against proteinase 3 , a serine proteinase located in azurophilic granules of human PMNL. ${ }^{3}$ Antiproteinase 3 antibodies may also occur in isolated, rapidly progressive glomerulonephritis and occasionally in systemic vasculitic conditions other than Wegener's granulomatosis. ${ }^{2}$ Antibodies directed against other lysosomal enzymes in PMNL azurophilic granules, for example myeloperoxidase and elastase, also occur in primary systemic vasculitic diseases and rapidly progressive glomerulonephritis. ${ }^{34}$ After ethanol fixation of PMNL, proteinase 3 remains within the cytoplasmic granules, whereas myeloperoxidase and elastase become extracted and locate close to or on the cell nucleus. ${ }^{2+}$ At indirect immunofluorescent microscopical determination of ANCA, anti-proteinase 3 antibodies produce a typical pancytoplasmatic granular staining pattern (C-ANCA), whereas antimyeloperoxidase and anti-elastase antibodies give rise to a blurry perinuclear staining pattern (P-ANCA) or staining of the PMNL nuclei (granulocyte specific anti-nuclear antibodies, GS-ANA). ${ }^{245}$ A detergent extract of isolated azurophil granules ( $\alpha$ antigen) can be used for the detection of anti-proteinase 3 antibodies (C-ANCA) by means of enzyme linked immunosorbent assay (ELISA) but does not allow detection of anti-myeloperoxidase. ${ }^{56}$ Also, antibodies against lactoferrin, an iron binding protein residing in specific granules of PNML, ${ }^{7}$ produce a P-ANCA pattern owing to perinuclear/nuclear localisation of lactoferrin after ethanol fixation. ${ }^{8}$ Apart from the artifactual GS-ANA staining pattern caused by antibodies directed against nucleophilic cytoplasmic antigens, it is possible that true GS-ANAs also exist.

Apart from the occurrence in primary systemic vasculitides and rapidly progressive glomerulonephritis, P-ANCA/GS-ANA may be seen in other disease states, for example rheumatoid arthritis without signs of vasculitis, ${ }^{10}$ inflammatory bowel disease, and primary sclerosing cholangitis. ${ }^{11-15}$ Recent evidence favours the idea that inflammatory bowel disease may be caused by mesenteric vasculitis. ${ }^{16-18}$

In a preliminary study of 16 frozen sera from patients with Crohn's disease we found low levels of anti- $\alpha$ antigen antibodies, anti-myeloperoxidase antibodies, and anti-lactoferrin antibodies in some sera. ${ }^{19}$ The present study was done to extend these observations and to include sera from patients with ulcerative colitis and primary sclerosing cholangitis.

\section{Patients and methods}

CROHN'S DISEASE

Fifty two patients, 27 men aged 21-71 years (mean 48 years) and 25 women aged $17-55$ years (mean 38 years) were enrolled in the study. The mean duration of the disease was 17 years (range 4-33 years). Eight patients had disease limited to the colon and/or rectum, 19 patients had disease in the small bowel alone, and 25 patients had disease manifestations in both small bowel and colon/rectum. Forty patients had been operated on, 14 of whom had been subjected to only ileocoecal resection. Three patients had ileorectal anastomosis, and six patients had ileostomy after proctocolectomy.

Twenty four patients, 11 men aged19-65 years 
$\lg G$ anti- $\alpha$ antigen

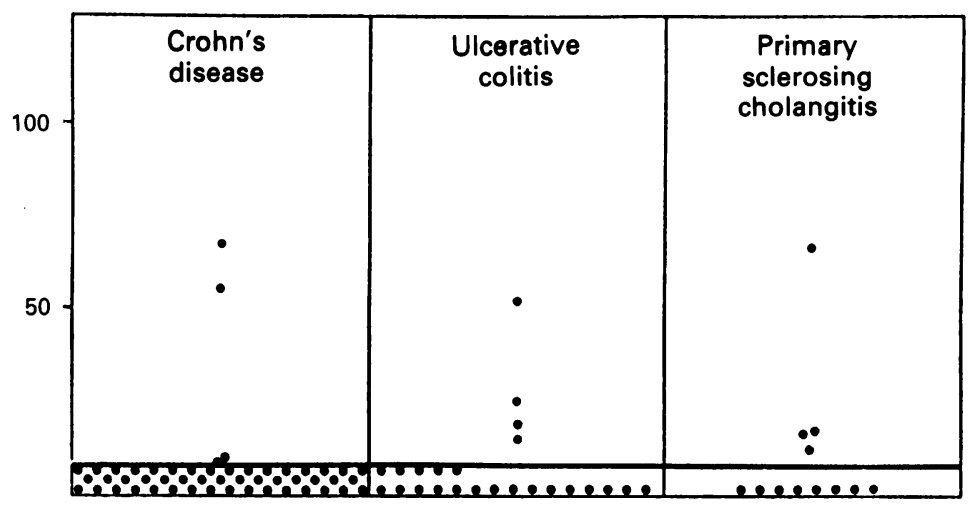

IgG anti-myeloperoxidase

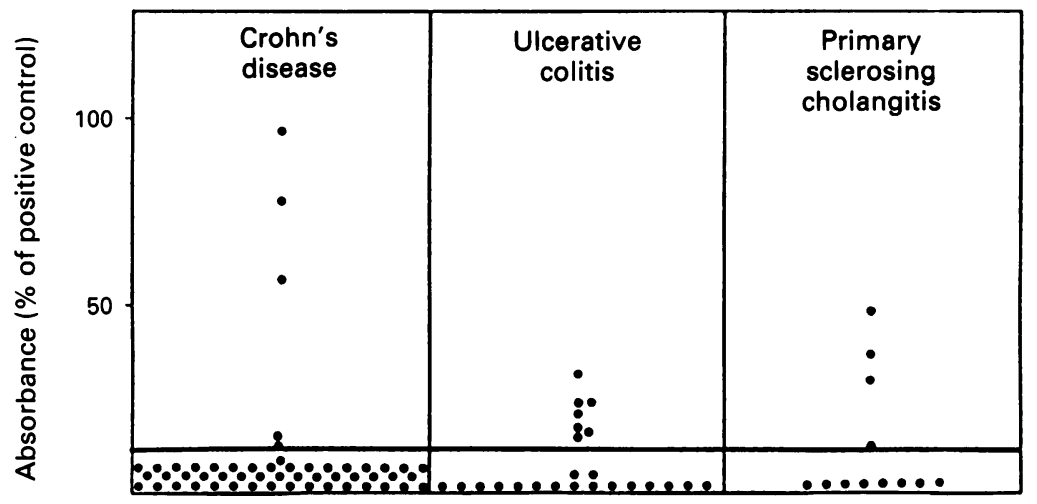

$\lg G$ anti-lactoferrin

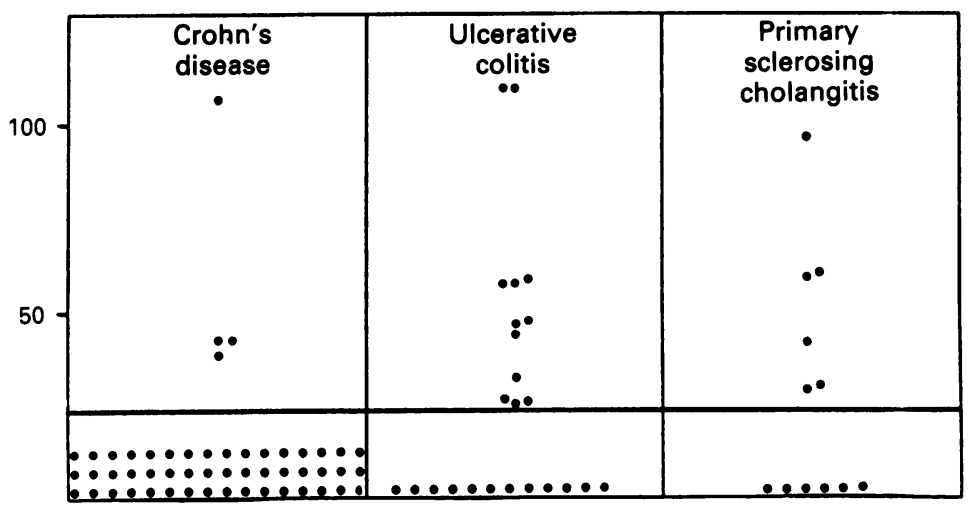

Figure 1: Diagram to illustrate the ELISA results of IgG autoantibody directed against $\alpha$ antigen, myeloperoxidase, and lactoferrin in sera from patients with Crohn's disease $(n=52)$, ulcerative colitis $(n=24)$, and primary sclerosing cholangitis $(n=12)$. The lower limit for positive ELISA (that is, $+2 S D$ of the mean value of 218 normal reference sera) is indicated.

(mean 46 years), and 13 women aged $20-68$ years (mean 42 years) participated in the study. Nineteen patients had left sided colitis and five patients had more extensive or total colitis. Fourteen of the patients had active disease - that is at least five loose stools with visible blood (all had visible inflammation at endoscopy) - and the remaining 10 patients were in clinical remission.

PRIMARY SCLEROSING CHOLANGITIS

Twelve patients, seven men aged 20-59 years (mean 38 years) and five women aged $35-63$ years (mean 48 years), took part in the study. The diagnosis had been made by endoscopic retrograde cholangiography 3 years (range 1-6 years) before the study. Two patients had only intrahepatic cholangitis and 10 had both extra- and intrahepatic cholangitis. In four patients cirrhosis of the liver had been diagnosed at histopathological examination of liver biopsies. One patient had undergone liver transplantation 6 years earlier. Nine of the 12 patients had also suffered from ulcerative colitis for 8 years (range 0-21 years) and one had non-specific colitis. In the two remaining patients no accompanying disease had been diagnosed. None of the patients had Crohn's disease.

One of the patients originally participating in the study had to be excluded as she proved to have suffered from acute Campylobacter jejuni colitis.

\section{ANTIGEN PREPARATIONS}

Azurophil granules were prepared from isolated normal peripheral granulocytes after low pressure homogenisation of the cells and centrifugation of the homogenate in a Percoll (Pharmacia Fine Chemicals, Uppsala, Sweden) gradient as described elsewhere. ${ }^{5}$ The granules were lysed with $0.01 \%$ (final concentration) Triton X-100 (Merck, Darmstadt, Germany). ${ }^{5}$ The extract thus achieved ( $\alpha$ antigen) was diluted in carbonate-bicarbonate buffer $\mathrm{pH} 9.5$ and used as substrate for ELISA. ${ }^{5}$

Lyophilised preparations of myeloperoxidase (Calbiochem, La Jolla, CA, USA), human milk lactoferrin (Sigma Chemical Co, St Louis, MO, USA), and bovine milk lactoferrin (Sigma) were dissolved in carbonate-bicarbonate buffer (10 $\mu \mathrm{g}$ antigen $/ \mathrm{ml}$ ) and used for ELISA.

\section{ELISA}

High binding plastic microtitre plates (Nunc Immunoplate, Roskilde, Denmark) were coated with $\alpha$ antigen, myeloperoxidase, or human lactoferrin solutions by incubation at $4^{\circ} \mathrm{C}$ for 12 hours. After washing with buffer (phosphate buffered saline, PBS, with $0.05 \%$ Tween 20 ), serum samples (diluted 1:10 in PBS-Tween) were applied for 30 minutes at room temperature. Normal human serum diluted 1:10 served as a blank. The microtitre plates were washed thoroughly with PBS-Tween and incubated for another 30 minutes with alkaline-phospatase (ALP) conjungated rabbit anti-human $\gamma$ chain or rabbit anti-human $\alpha$ chain antisera (Dako, Glostrup, Denmark) diluted 1:400 in PBSTween. After washing, the substrate buffer was applied and the optical density (OD) read at $405 \mathrm{~nm}$ when the positive reference samples had reached OD $1 \cdot 0$. OD values exceeding 2 SDs of the reference material from healthy blood donors $(\mathrm{n}=218$ for IgG tests; $\mathrm{n}=211$ for IgA tests) were considered positive. The IgG autoantibody analyses were performed on all sera from patients with Crohn's disease $(n=52)$, ulcerative colitis $(n=24)$, and primary sclerosing cholangitis $(n=12)$. IgA autoantibody tests were done on sera from 51 patients with Crohn's disease, 21 ulcerative colitis sera, and 11 sera from patients with primary sclerosing cholangitis. 


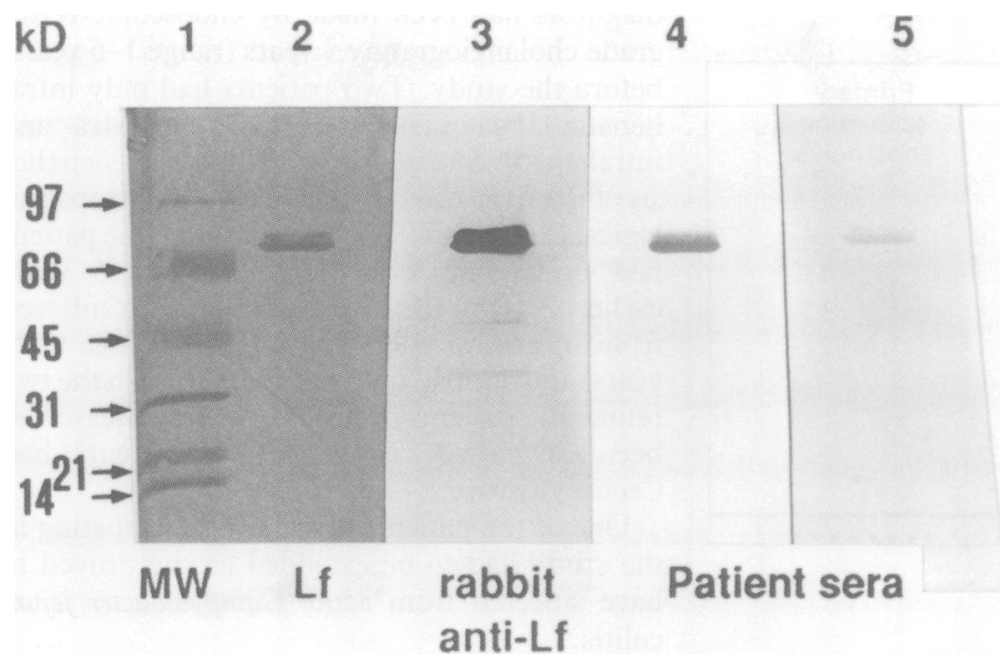

Figure 2: Sodium dodecyl sulphate polyacrylamide gels (SDS-PAGE) electrophoresis of the human milk lactoferrin preparation (lane 1) showed only material of molecular weight about 80 $k D$ when stained with Comassie blue. Lane 2 shows molecular weight markers for comparison. Immunoblotting, using polyclonal rabbit anti-human leukocyte lactoferrin, showed slight

reaction with antigens of lower molecular weight, apart from the strong reaction with the $80 \mathrm{kD}$ lactoferrin band (lane 3). Patient sera positive for IgG anti-human milk lactoferrin, as tested by ELISA, however, reacted only with lactoferrin.

IgG anti-bovine lactoferrin antibodies were measured essentially as described above, except that no negative or positive serum references were used. OD values were read after 30 minutes incubation with substrate buffer. Eleven positive and 10 negative sera in the test for IgG antihuman lactoferrin were selected for this analysis.

\section{RABBIT ANTI-LACTOFERRIN ANTISERA}

Serum containing polyclonal rabbit anti-human leukocyte lactoferrin was the kind gift of Dr Johan Richter, Department of Internal Medicine, University Hospital, Lund, Sweden. To produce anti-human milk lactoferrin antibodies, rabbits were immunised by subcutaneous injections of $2 \mathrm{mg}$ human milk lactoferrin (that is, the same antigen as that used for the ELISA and western blot assays) together with Freund's incomplete adjuvant (Sigma) on three occasions with 4 week intervals. The reason for not using Freund's complete adjuvant was to avoid immunisation against hsp-65, because of its immunological cross reaction with human lactoferrin.$^{20}$ Two weeks after the second booster dose, the animals were anaesthetised with barbiturate, and exsanguinised by heart puncture. Serum was pooled. Anti-lactoferrin activity of the serum was confimed by agarose double radial immunodiffusion against human milk lactoferrin.

\section{WESTERN BLOTTING}

Lactoferrin ( $1 \mu \mathrm{g}$ per lane) was electrophoresed in $5-20 \%$ sodium dodecyl sulphate polyacrylamide gels (SDS-PAGE) and transferred to polyvinylidene difluoride membranes (Millipore Continental Water Systems, Bedford, MA, USA). Blocking was done with $5 \%$ bovine serum albumin (Sigma) in PBS. Anti-lactoferrin positive patient sera (as judged by ELISA) were diluted $1: 20$ or $1: 200$ in PBS-Tween with $1 \%$ bovine serum albumin, and applied for 1 hour.
The rabbit anti-human leukocyte lactoferrin served as a reference. ALP conjugated antihuman IgG and anti-rabbit IgG (Dako) were used as secondary antibodies.

\section{INDIRECT IMMUNOFLUORESCENCE (IIF)}

MICROSCOPY

To study the distribution of lactoferrin in ethanol fixed granulocytes cytocentrifuged onto microscope slides, the slides were incubated with rabbit anti-human milk lactoferrin in a moist chamber for 30 minutes. After washing with PBS and incubation for another 30 minutes with sheep fluorescein isothiocyanate (FITC) conjugated anti-rabbit Ig (Wellcome Diagnostics, Temple Hill, Dartford, UK), the slides were again washed with PBS, mounted with PBSglycerin, and inspected under a fluorescence microscope with a mercury lamp (HBO 50) epiillumination and filters for FITC activation/ emission.

\section{STATISTICS}

Differences in ELISA results between the control sera and the patient sera were evaluated by the $\chi^{2}$ test, and divided into four groups: $p \geq 0 \cdot 05$ $=$ not significant (NS); $<00.05\left({ }^{\star}\right) ; \mathrm{p}<0.01(\star \star)$; $\mathrm{p}<0.001(\star \star \star)$.

\section{Results}

The frequencies of IgG antibodies directed against $\alpha$ antigen, myeloperoxidase, and lactoferrin are shown in Figure 1.

In Crohn's disease 4 of 52 sera had IgG anti- $\alpha$ antigen, which is not statistically different from the control group, although two of the sera had remarkably high antibody levels. In ulcerative colitis 4 of 24 of the sera contained IgG anti- $\alpha$ antigen antibodies $(p<0.05)$, and in primary sclerosing cholangitis 4 of 12 sera $(p<0.001)$ were positive.

IgG anti-myeloperoxidase antibodies occurred in 3 of 52 of the sera taken from patients with Crohn's disease (not statistically different from

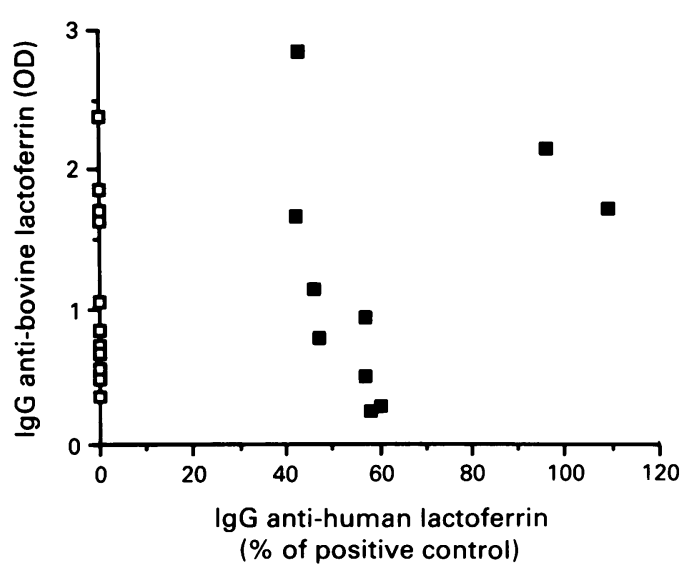

Figure 3: Diagram to illustrate the lack of correlation between ELISA results for IgG anti-human lactoferrin

autoantibodies and IgG antibodies directed against bovine milk lactoferrin. Ten patient sera positive in the ELISA for IgG anti-human lactoferrin ( $\square$ ) and 12 randomly selected normal reference sera ( $\square$ ) negative in the IgG anti-human lactoferrin test were analysed. 
$\lg \mathrm{A}$ anti- $\alpha$ antigen

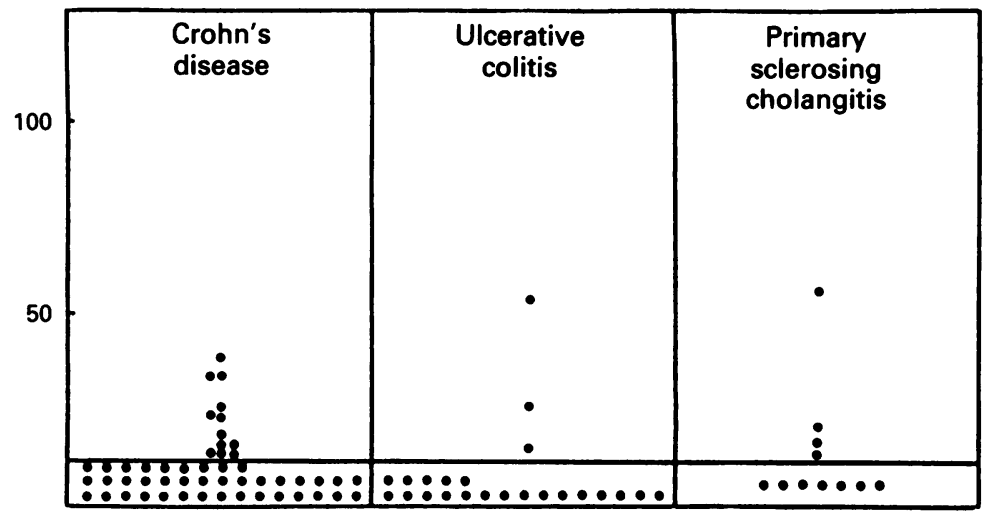

IgA anti-myeloperoxidase

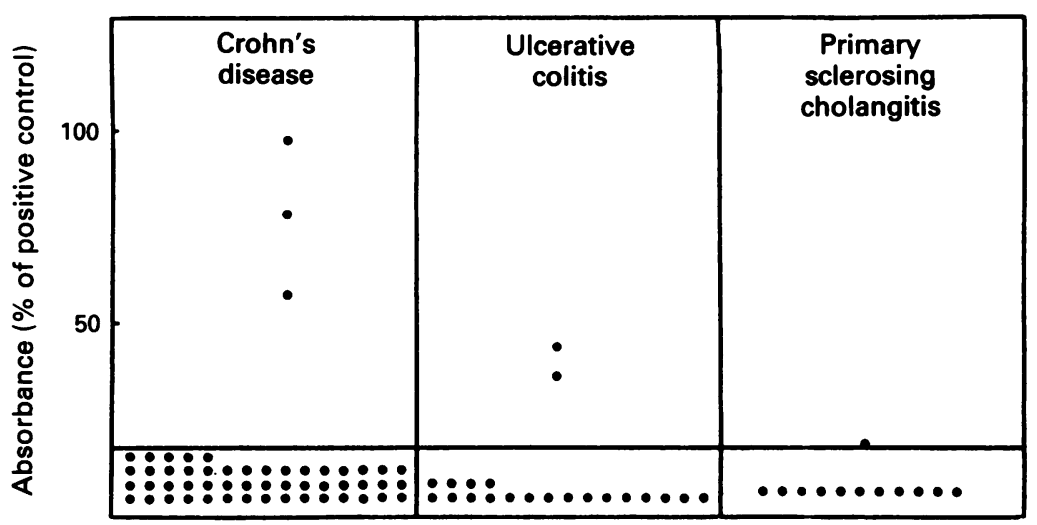

$\lg \mathrm{A}$ anti-lactoferrin

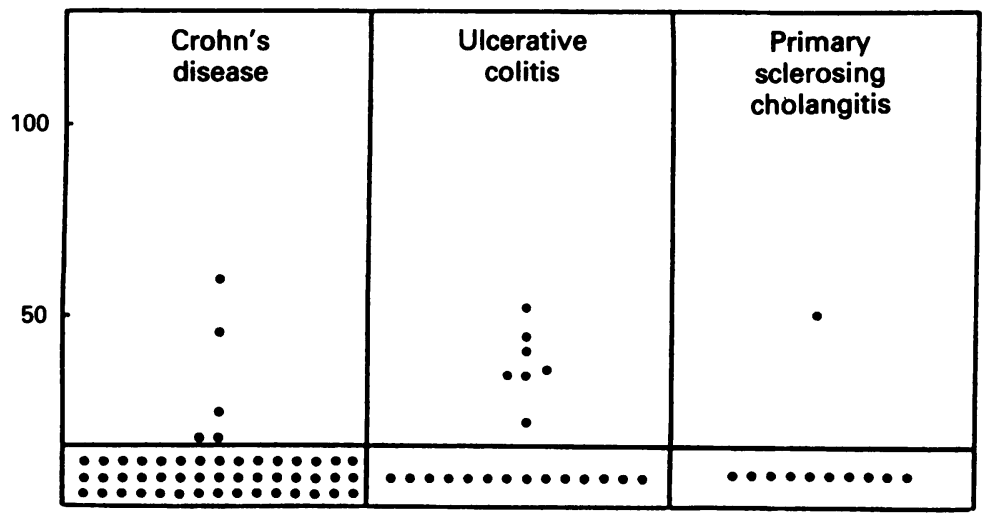

Figure 4: Results of the ELISA tests for IgA autoantibodies directed against $\alpha$ antigen, myeloperoxidase, and lactoferrin. Fifty one sera from patients with Crohn's disease, 21 sera from patients with ulcerative colitis, and 11 sera from patients with primary sclerosing cholangitis were analysed. The cut of levels (that is, values exceeding $2 S D$ of the mean of a reference material of 211 normal sera) are indicated.

the control group), and in 7 of 24 of the ulcerative colitis sera $(p<0 \cdot 001)$. Four of 12 sera from patients with primary sclerosing cholangitis were positive $(\mathrm{p}<0 \cdot 001)$.

Four of $52(8 \%)$ of the sera from patients with Crohn's disease contained IgG anti-lactoferrin antibodies (not significantly different from the control group). All of these sera proved to come from patients with Crohn colitis. However, colitis also occurred in 33 of $48(69 \%)$ without IgG anti-lactoferrin antibodies. Both in ulcerative colitis and primary sclerosing cholangitis,
IgG anti-lactoferrin antibodies were found in $50 \%$ of the sera $(p<0.001$ in both instances). All of the anti-lactoferrin positive cholangitis sera belonged to patients suffering from concomitant ulcerative colitis - that is, 6 of $9(67 \%)$ sera from patients with sclerosing cholangitis and concomitant ulcerative colitis contained IgG antilactoferrin antibodies. Two of the sera from patients with primary sclerosing cholangitis and one serum from a patient with ulcerative colitis were positive in all three ELISA tests. However, in all three instances the ELISA tests gave quite different OD values in the different assays, indicating true positive ELISA test results.

Positive anti-lactoferrin ELISA results were blocked by preincubation of the lactoferrincoated microtitre plates with rabbit IgG antilactoferrin (not illustrated). The occurrence of IgG anti-lactoferrin was also confirmed by western blotting, revealing a single reaction band with an antigen of molecular weight about 80000 and corresponding to rabbit anti-human leukocyte lactoferrin (Fig 2).

One patient originally included in the study had acute colitis and a high level of IgG antilactoferrin at the first sampling occasion, and a further raised anti-lactoferrin level in a later serum sample (not illustrated). This patient was, however, excluded from the study since her symptoms were explained by infection with Campylobacter jejuni.

Figure 3 illustrates the lack of correlation between levels of IgG anti-human lactoferrin and IgG anti-bovine lactoferrin as tested by ELISA with 10 sera positive in the anti-human lactoferrin and 12 sera negative in the same test.

Figure 4 shows the occurrence of IgA antibodies directed against $\alpha$ antigen, myeloperoxidase, and human lactoferrin.

Raised levels of $\operatorname{IgA}$ anti- $\alpha$ antigen were seen in 12 of 51 (24\%) sera from Crohn's disease $(\mathrm{p}<0.001)$, in 5 of $11(45 \%)$ sera from patients with primary sclerosing cholangitis $(p<0.001)$, but in only 2 of $21(10 \%)$ sera from patients with ulcerative colitis (NS). The levels of IgA antimyeloperoxidase did not differ from the control group in any of the disease states, whereas IgA anti-lactoferrin was significantly $(p<0.05)$ more common in Crohn's disease, although it was seen only in a minority of the patient sera $(5$ of $51=$ $10 \%)$. IgA anti-lactoferrin was found in significantly $(p<0.001)$ increased frequency also in ulcerative colitis ( 7 of $21=33 \%$ ), but not in sclerosing cholangitis ( 1 of $11=9 \%$ ).

The levels of IgA-ANCAs were not explained by the occurrence of agglutinating rheumatoid factors (not illustrated).

Figure 5 shows the frequency of positive IgG and/or IgA tests for either anti- $\alpha$ antigen, or antimyeloperoxidase, or anti-lactoferrin in sera from blood donors, Crohn's disease, ulcerative colitis, and primary sclerosing cholangitis. Taken together, positive IgG tests for one or more of the three granulocyte antigens were found in $11 \%$ (24 of 218) control sera, in 19\% (10 of 52) of Crohn's disease (NS), in 67\% (16 of 24) of ulcerative colitis ( $p<0.001$ ), and in $67 \%$ ( 8 of 12 ) of primary sclerosing cholangitis sera; IgA antigranulocyte antibodies were found in $7 \%$ (15 of 211 ) of the controls, in $29 \%$ ( 15 of 51) of Crohn's 


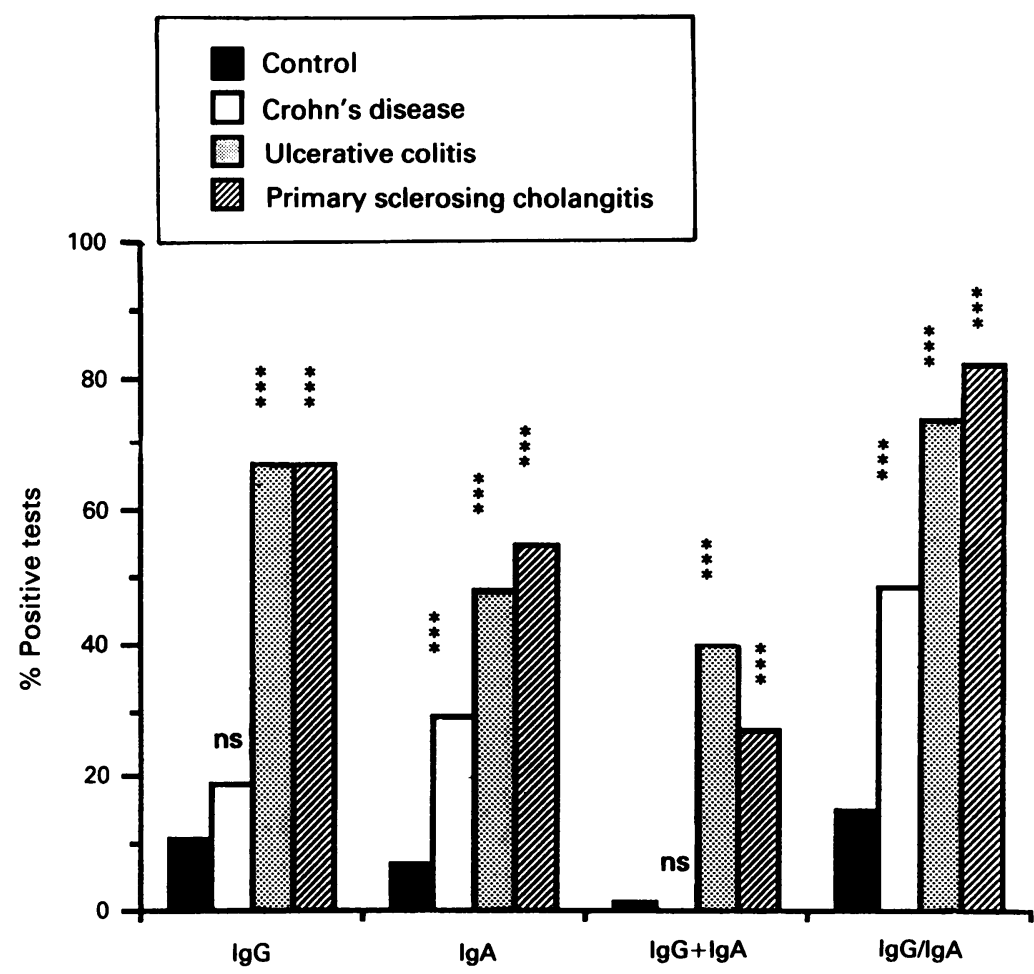

Figure 5: Diagram summarising the ELISA tests for IgG and/or IgA autoantibodies against all granulocyte antigens tested - that is, $\alpha$ antigen, myeloperoxidase, and human lactoferrin.

disease patients $(\mathrm{p}<0.001)$, in $48 \%$ (10 of 21$)$ of ulcerative colitis patients $(\mathrm{p}<0.001)$, and in $55 \%$ (6 of 11) of primary sclerosing cholangitis subjects $(\mathrm{p}<0.001)$. Antibodies of either IgG or IgA class were found in $49 \%$ of the sera from patients with Crohn's disease $(p<0.001)$, in $74 \%$ of the sera from patients with ulcerative colitis $(\mathrm{p}<0.001)$, and in $82 \%$ of the cholangitis sera $(\mathrm{p}<0.001)$.

There was no obvious correlation between disease activity or extraintestinal disease (arthritis, arthralgia, skin involvement) and the presence of IgG or IgA anti-granulocyte

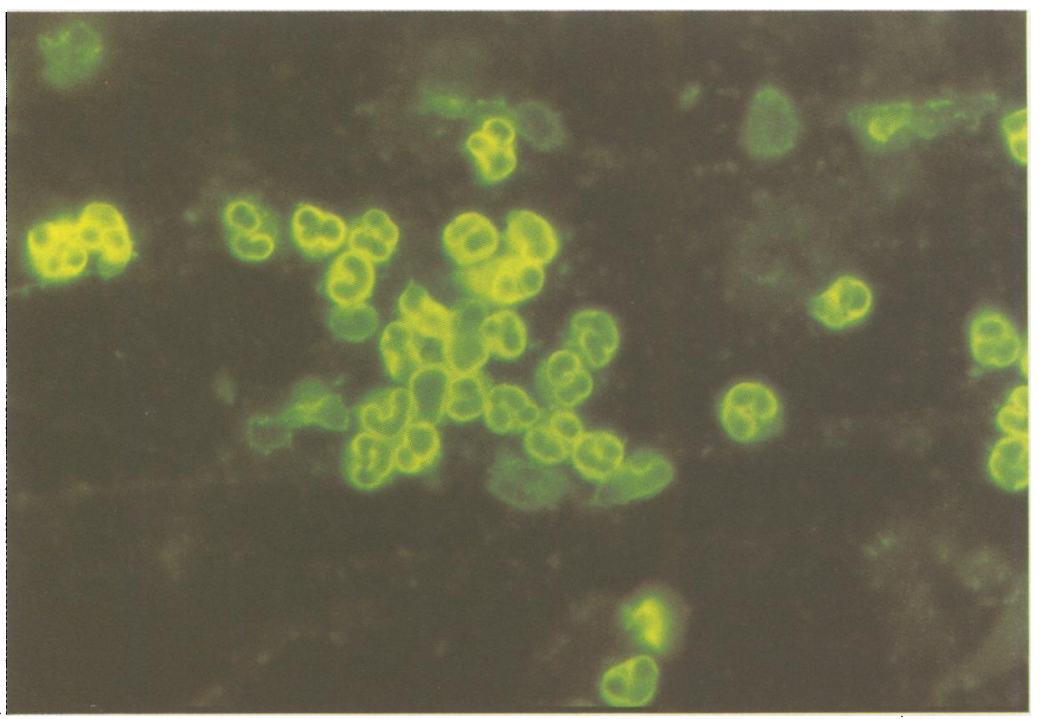

Figure 6: Granulocyte specific antinuclear antibodies/perinuclear staining anti-neutrophil cytoplasm antibodies (GS-ANA/P-ANCA/IIF staining pattern achieved after incubation of anti-human milk lactoferrin antibodies with cytocentrifuged, ethanol fixed human polymorphonuclear granulocytes. antibodies. The levels of IgG and IgA antigranulocyte antibodies did not correlate to the total serum levels of IgG and IgA (not illustrated).

Figure 6 shows the GS-ANA/P-ANCA immunofluorescence pattern of cytocentrifuged ethanol-fixed human polymorphonuclear neutrophil granulocytes after staining with rabbit anti-human milk lactoferrin. This, taken together with the western blot analysis (Fig 2), shows that antibodies against human milk lactoferrin recognise human leukocyte lactoferrin and vice versa.

\section{Discussion}

In this study we report high frequencies of IgG anti-lactoferrin antibodies (corresponding to P-ANCA) in sera from patients with ulcerative colitis and primary sclerosing cholangitis. In Crohn's disease, however, serum anti-lactoferrin antibodies of IgG were rarely detected, and then only in patients with colonic disease manifestations. IgA anti-lactoferrin antibodies were, however, found in some cases of both ulcerative colitis and Crohn's disease.

Several different types of autoantibodies have been described in inflammatory bowel disease and primary sclerosing cholangitis. ${ }^{11-15} 1921-27$ Although none of the autoantibodies have been proved to be of pathogenetic significance, it has been shown, both in Crohn's disease and in ulcerative colitis, that IgG and complement can be present on the apical surface of enterocytes in vivo, ${ }^{28} 29$ possibly indicating a pathogenetic role for anti-epithelial/anti-brush border antibodies in inflammatory bowel disease. In this connection, and considering the high frequency of antilactoferrin antibodies found in the present study, it is interesting to note that lactoferrin has been reported to bind to intestinal brush border via a specific receptor. ${ }^{30}$ Antibodies of IgA class directed against Saccharomyces cerviciae are common in Crohn's disease but not in ulcerative colitis, and determination of such antibodies may be of diagnostic help. ${ }^{31}$ It is possible that many of the anti-microbial antibodies described in inflammatory bowel disease and other inflammatory disease states, may in fact be reflections of immunisation against microbial heat-shock proteins, which has been implied in the pathogenesis of several autoimmune disease states $^{32}$ and which may induce anti-lactoferrin antibodies $^{22}$ (see below).

Wakefield et al presented evidence that vasculitis and microthrombosis in mesenteric vessels can be important pathogenetic factors in inflammatory bowel disease. ${ }^{16-18}$ Furthermore, P-ANCA/GS-ANA, which occurs in several primary vasculitis diseases, can frequently be demonstrated in inflammatory bowel disease and primary sclerosing cholangitis also. ${ }^{11-15} \mathrm{P}$-ANCA /GS-ANA is more common in ulcerative colitis than in Crohn's disease, ${ }^{113-15}$ which is confirmed in the present study by the high frequency of IgG anti-lactoferrin antibodies in ulcerative colitis and primary sclerosing cholangitis but not in Crohn's disease. In Crohn's disease the frequency. of IgG anti- $\alpha$ antigen and antimyeloperoxidase antibodies did not differ 
statistically from the control group, although in a few instances the antibody levels were surprisingly high. In both ulcerative colitis and primary sclerosing cholangitis the frequency of positive IgG anti- $\alpha$ /anti-myeloperoxidase tests differed statistically from the control group, but in general the levels were low and, considering the small number of patients with positive anti- $\alpha /$ anti-myeloperoxidase tests, the relevance of these results is uncertain. However, it is interesting that these types of ANCAs were found at all, since they are considered to be reliable markers of primary systemic vasculitis. ${ }^{2}$ ANCAs of IgA class have been reported in Hennoch-Schönleins purpura and IgA nephropathy, although these findings are sometimes explained by the presence of rheumatoid factor..$^{33}$ The appearance of antibodies directed against $\alpha$ antigen in some cases of ulcerative colitis (IgG) and in Crohn's disease (IgA) could possibly favour the hypothesis of primary vasculitis as a pathogenetic factor in these diseases. ${ }^{16-18}$

Lactoferrin is an iron binding protein that occurs abundantly not only in the specific granules of granulocytes, ${ }^{7}$ but also in tears, milk, and secretions at mucosal surfaces. ${ }^{34-36}$ Raised levels of circulating lactoferrin are seen during active inflammatory disease. ${ }^{37}$ It exerts antibacterial effects by depriving bacteria of iron required for growth, and it has anti-inflammatory properties - for example, by preventing complement activation through inhibition of classical C3 convertase. ${ }^{38}$ Lactoferrin can also prevent the formation of hydroxyl radicals by iron binding. ${ }^{36-39}$ Lactoferrin may thus be of great importance as a non-specific anti-phlogistic defence factor at the primary immunological barriers. It has been shown experimentally that binding of lactoferrin by anti-lactoferrin antibodies increases the amount and duration of hydroxyl radical formation by granulocytes. ${ }^{39}$ Hypothetically, anti-lactoferrin autoantibodies could, by counteracting the anti-inflammatory effects of lactoferrin, aggravate and prolong mucosal inflammation induced by several different mechanisms, and the antibodies may therefore have pathogenetic significance even though their occurrence does not seem to correlate with disease activity. Anti-lactoferrin antibodies may also have pathogenetic effects by activation of primed granulocytes infiltrating the gut mucosa or adhering to vessel walls, in analogy with the effects of other types of ANCA. ${ }^{54041}$ In addition, mucosal lactoferrin/anti-lactoferrin complexes may stimulate intestinal goblet cells to excessive mucus secretion, as do other immune complexes. ${ }^{42}$ On the other hand, the occurrence of anti-lactoferrin autoantibodies may, of course merely be an epiphenomenon without pathogenetic significance. The fact that the individual types of ANCA were found only in a minority $(\leq 50 \%)$ of the sera tested is an argument in favour of this. On the other hand, consumption of circulating antibodies cannot be ruled out in the seronegative instances.

The origin of anti-lactoferrin autoantibodies is unknown. Although bovine and human lactoferrin have molecular and antigenic similarities, ${ }^{35}$ immunisation of experimental animals with bovine lactoferrin seldom seems to result in cross immunisation against human lactoferrin, ${ }^{3+}$ and the lack of correlation between levels of antihuman lactoferrin and anti-bovine lactoferrin shown in this study contradicts the hypothesis that cross immunisation against dietary bovine lactoferrin explains the appearance of antihuman lactoferrin autoantibodies. An exciting possibility is the antigenic mimicry between the $65 \mathrm{kD}$ mycobacterial heat-shock protein (hsp65) and human lactoferrin, and the fact that immunisation against hsp-65 results in the production of anti-lactoferrin antibodies. ${ }^{32}$ Mycobacterial infection and immunisation against mycobacterial antigens/hsp-65 have been implicated in several autoimmune disease states, both experimental and clinical..$^{2031+3-45}$ Considering the occurrence of $\operatorname{IgA}$ anti-lactoferrin antibodies in some cases of Crohn's disease in the present study, we find it interesting that IgA (but not IgG) anti-hsp-65 antibodies have been reported in Crohn's disease. ${ }^{42}$ Furthermore, the registration of anti-lactoferrin antibodies in a case of Campylobacter colitis in this study is interesting with regard to the possibility of cross immunisation against microbial antigen(s). We therefore intend to analyse additional sera from patients with infectious gastroenteritis for the presence of anti-lactoferrin antibodies.

In conclusion, we have shown high frequencies of IgG anti-lactoferrin antibodies in ulcerative colitis, and primary sclerosing cholangitis, but not in Crohn's disease, whereas IgA autoantibodies directed against lactoferrin were found in some cases of both ulcerative colitis and Crohn's disease. We suggest that anti-lactoferrin may be of pathogenetic significance by counteracting the antiphlogistic properties of lactoferrin at mucosal surfaces, thereby aggravating and/or sustaining mucosal inflammation initiated by other factors.

This study was financed by grants from Professor Nanna Svatrz Foundation, the Swedish Association against Rheumatism, King Gustaf Vth 80-Year Foundation, and the Swedish Medical Research Council.

I van der Woude FJ, Rasmussen N, Lobatto S, et al. Autoantibodies against neutrophils and monocytes: tool for diagnosis and marker of disease activi

2 Kallenberg CGM, Cohen Tervaert JW, van der Woude FJ, et al. Autoimmunity to lysosomal enzymes: a new clue to vasculitis and glomerulonephritis? Immunol Today 1991; 12: $61-4$

3 Jenne DE, Tschopp J, Lüdemann J, Utecht B, Gross WL. Wegener's auto-antigen decoded. Nature 1990; 346: 520 .

4 Falk RJ, Jenette JC. Anti-neutrophil-cytoplasmic autoantibodies with specificity for myeloperoxidase in patients with systemic vasculitis and idiopathic necrotizing and with systemic vasculitis and idiopathic necrotizing and crescentic

5 Skogh T, Dahlgren C, Holmgren K, et al. Anti-granulocyte antibodies (C-ANC, P-ANCA, GS-ANA) studied by confocal scanning laser fluorescence microscopy, ELISA, and focal scanning laser fluorescence microscopy, ELISA, and
chemiluminescence techniques. Scand F Immunol 1991; 34: 137-45.

6 Rasmussen N, Siölin C, Isaksson B, et al. An ELISA for the detection of anti-neutrophil cytoplasm antibodies (ANCA). f Immunol Meth 1990; 127: 139-45.

7 Furmanski P, Li Z-P. Multiple forms of Lactoferrin in Normal and Leukemic Human Granulocytes. Exp Hematol 1990; 18: 932-5.

8 Briggs RC, Glass II WF, Montiel MM, Hnilica LS. Lactoferrin: nuclear localization in the human neutrophilic granulocyte? 7 Histochem Cytochem 1981; 29: 1128-36.

9 Wiik A. Granulocyte-specific antinuclear antibodies. Allergy $1980 ; 35: 263-89$.

10 Wiik A, Munthe E. Complement fixing granulocyte-specific antinuclear factors in neutropenic cases of rheumatoid antinuclear factors in neutropenic cases

11 Nielsen H, Wiik A, Elmgreen J. Granulocyte specific antinuclear antibodies in ulcerative colitis. Aid in differential 
diagnosis of inflammatory bowel disease. Acta Pathol Microbiol Scand [Sect C] 1983; 91: 23-6.

12 Snook JA, Chapman RW, Fleming K, Jewell DP. Antineutrophil nuclear antibody in ulcerative colitis, Crohn's disease and primary sclerosing cholangitis. Clin Exp Immunol 1989; 76: 30-3.

13 Saxon A, Shanahan F, Landers C, et al. A distinct subset of antineutrophil cytoplasmic antibodies is associated with inflammatory bowel disease. $\mathcal{F}$ Allergy Clin Immunol 1990; 86: 202-10.

14 Duerr RH, Targan SR, Landers CJ, et al. Anti-neutrophil cytoplasmic antibodies in ulcerative colitis. Gastroenterology cytoplasmic antibodic.

15 Duerr RH, Targan SR, Landers CJ, et al. Neutrophil cytoplasmic antibodies: a link between primary sclerosing cholangitis and ulcerative colitis. Gastroenterology 1991; 100 ;: 1385-91

16 Wakefield AJ, Sawyerr AM, Dhillon AP, et al. Pathogenesis of Crohn's disease: multifocal gastrointestinal infarction. Lancet 1989; ii: 1057-62.

17 Sawyerr AM, Wakefield AJ, Rowles P, Dhillon AP, Pittilo M, Hudson M, et al. Microvascular changes in Crohn's disease
and ulcerative colitis: examination by vascular casting and scanning electron microscopy. Gut 1990; 31 : A623.

18 Wakefield AJ, Sankey EA, Dhillon AP, et al. Granulomatous vasculitis in Crohn's disease. Gastroenterology 1991; 100:

19 Peen E, Tejle K, Skogh T. Anti-granulocyte antibodies in Crohn's disease. APMIS 1990; suppl 19: 34

20 Esaguy N, Aguas AP, van Embden JDA, Silva MT. Mycobacteria and human autoimmune disease: direct evidence of cross-reactivity between human lactoferrin and the $65-$
kilodalton protein of tubercle and leprosy bacilli. Infect Immun 1991; 59: 1117-25.

21 Broberger O, Perlmann P. Autoantibodies in human ulcerative colitis. F Exp Med 1959; 110: 657-74.

22 Perlmann P, Hammarström S, Lagercrantz R, Campbell D. Autoantibodies to colon in rats and human ulcerative colitis: cross reactivity with Escherichia coli O:14 antigen. Proc Soc Exp Biol Med 1967; 125: 975-80.

23 Das KM, Kadono Y, Fleischner GM. Antibody-dependent cell-mediated cytotoxicity in serum samples from patients with ulcerative colitis. Am $\mathcal{F}$ Med 1984; 77: 791-6.

24 Skogh T, Heuman R, Tagesson C. Anti-brush border antibodies (ABBA) in Crohn's disease. 7 Clin Lab Immunol 1982; 9: 147-50.

25 Skogh T, Bodemar G, Kihlström E, Ljunghusen O. Antibrush border antibodies (ABBA) in sera from patients with
ulcerative proctocolitis and in sera with antibodies against Yersinia enterocolitica O:3. F Clin Lab Immunol 1986; 19: 117-8.

26 Chapman RW, Cottone M, Selby WS, et al. Serum autoantibodies, ulcerative colitis and primary sclerosing cholangitis. Gut 1986; 27: 86-91.

27 Snook JA, Lowes JR, Wu KC, et al. Serum and tissue antibodies to colonic epithelium in ulcerative colitis. Gut 1991; 32: 163-6.

28 Green FHY, Fox H. The distribution of mucosal antibodies in the bowel of patients with Crohn's disease. Gut 1975; 16: 125-31.

29 Hallstensen TS, Mollnes TE, Garred P, et al. Epithelial deposition of immunoglobulin Gl and complement (C3b and terminal complement complex) in ulcerative colitis. Gastroenterology 1990; 98: 1264-71.

30 Davidsson L, Lönnerdal B. Specific binding of lactoferrin to brush-border membrane. Am f Physiol 1988; 254: G580-5.

31 Barnes RMR, Allan S, Taylor-Robinson $\mathrm{CH}$, et al. Serum antibodies to Saccharomyces servisiae in inflammatory bowel antibodies to Saccharomyces servisiae in inflammatory bowel disease: Is IGA antibody a marker for Croh

32 Winfield J, Jarjour W. Do stress proteins play a role in arthritis and autoimmunity? Immunol Rev 1991; 121: 193-220.

33 Daha MR, Falk RJ. Ig-class specificity of ANCA. Neth f Med 1990; 36: 126-7.

34 Wang C-S, Chan W-Y, Kloer UH. Comparative studies on the chemical and immunochemical properties of human milk, human pancreatic juice, and bovine milk lactoferrin. Comp Biochem Physiol [B] 1984; 78: 575-80.

35 Magnuson JS, Henry JF, Yip T-T, Hutchens TW. Structural homology of human, bovine, and porcine milk lactoferrins: evidence for shared antigenic determinants. Pediatr Res 1990; 28: 176-81.

36 Kuizenga A, van Haeringen NJ, Kijlstra A. Inhibition of hydroxyl radical formation by human tears. Invest Ophthalmol Vis Sci 1987; 28: 205-13.

37 Adeyami EG, Campos LB, Loizou G, et al. Plasma lactoferrin and neutrophil elastase in rheumatoid arthritis and systemic and neutrophil elastase in rheumatoid arthritis and systemi

38 Kievits F, Kijlstra A. Inhibition of C3 deposition on solidphase bound immune complexes by lactoferrin. Immunology 1985; 54: 449-57.

39 Britgan BE, Hassett DJ, Rosen GM, et al. Neutrophil degranulation inhibits potential hydroxyl radical formation. Biochem 7 1989; 264: 447-55.

40 Falk RJ, Terrell RS, Charles LA, Jenette JC. Anti-neutrophil cytoplasmic autoantibodies induce neutrophils to degranulate and produce oxygen radicals in vitro. Proc Natl Acad Sci USA 1990; 87: 4115-9.

41 Charles LA, Caldas MLR, Falk R, Terell RS, Jenette JC Antibodies against granule proteins activate neutrophils in Antibodies against granule proteins acti
vitro. F Leukoc Biol 1991; 50: 539-46.

42 Walker WA, Wu M, Bloch KI. Stimulation by Immune Complexes of Mucus Release from Goblet Cells of the Rat Complexes of Mucus Release from Goblet

43 Lydyard PM, Rook GA, Tsoulfa G, Sharif M, Smith M. Is Lydyard PM, Rook GA, Tsoulfa G, Sharif $M$, Smith $M$. Is
there a role for mycobacteria in the etiopathogenesis of rheumatoid arthritis? Immunol Rev 1991; 121: 137-54.

44 Shoenfeld Y, Isenberg DA. Mycobacteria and autoimmunity. Immunol Today 1988; 9: 178-82.

45 Hampson SJ, McFadden JJ, Hermon-Taylor J. Mycobacteria and Crohn's disease. Gut 1988; 29: 1017-9. 